\title{
LOS TARAYALES DE LA DEPRESION DEL EBRO
}

\author{
Federico FERNANDEZ-GONZALEZ, Andrés MOLINA \& Javier LOIDI
}

RESUMEN: La revisión de las comunidades de Tamarix en la depresión del Ebro permite reconocer tres asociaciones: Tamaricetum gallicae Br.-B1. \& O. Bolòs 1957 (Tamaricion africanae Br.-Bl. \& O. Bolòs 1957), riparia y subhalófila; Agrostio-Tamaricetum canariensis Cirujano 1981 (Tamaricion boveano-canariensis Izco, F. Fernández-González \& A. Molina 1984), mesohalófila (subass. tamaricetosum canariensis) o hiperhalófila (subass. inuletosum crithmoidis nova); y Suaedo braun-blanquetii-Tamaricetum boveanae (Izco \& al. 1984) ass. nova (Tamaricion boveano-canariensis), hiperhalófila y restringida a la parte más baja y oriental de la depresión.

Palabras clave: Tamarix, Nerio-Tamaricetea, España, fitosociología.

SUMMARY: Tamarix-communities belonging to the order Tamaricetalia (class NerioTamaricetea) are phytosociologically reviewed in the Ebro basin. Three associations can be differentiated: Tamaricetum gallicae Br.-Bl. \& O. Bolòs 1957 (Tamaricion africanae Br.-Bl. \& O. Bolòs 1957), riparian and subhalophilous; Agrostio-Tamaricetum canariensis Cirujano 1981 (Tamaricion boveano-canariensis Izco, F. Fernández-González \& A. Molina 1984), mesohalophilous (subass. tamaricetosum canariensis) or hiperhalophilous (subass. inuletosum crithmoidis nova); and Suaedo braun-blanquetii-Tamaricetum boveanae (Izco \& al. 1984) ass. nova (Tamaricion boveano-canariensis), hiperhalophilous and restricted to eastern and lower part of the basin.

Key words: Tamarix, Nerio-Tamaricetea, Spain, phytosociology.

\section{N T R O D U C C I O N}

Las especiales condiciones edáficas y climáticas de la depresión del Ebro permiten el desarrollo de abundantes formaciones arborescentes de carácter higrófilo y exigencias halófilas, presididas por especies del género Tamarix, que localmente reciben el nombre de tamarizales. Braun-Blanquet \& O. Bolòs (1957) abordaron el estudio parcial de estas comunidades aragonesas -excluyendo explícitamente los tarayales marcadamente halófilos- y establecieron las bases de su estructuración sintaxonómica. Recientemente Izco, Fernández-González \& Molina (1984) han propuesto una reestructuración del orden Tamaricetalia en la península Ibérica, fundamentada en la revisión de las tablas disponibles y el análisis de las comunidades murciano-almerienses y aragonesas (Salada de Chiprana) de Tamarix boveana. Con el presente trabajo se pretende completar el estudio de este tipo de vegetación en la 
depresión del Ebro, utilizando como hipótesis de partida las propuestas conceptuales de los últimos autores citados.

En este sentido, conviene distinguir tres tipos principales de situaciones topográficas e hidrológicas en las que prosperan los tarayales, y que han sido consideradas durante el muestreo fitosociológico de estas comunidades:

A) Tarayales de riberas de grandes ríos sobre depósitos gravosos, arenosos o limo-arenosos, con bajos niveles de salinidad (fig. 1A).

B) Tarayales de cauces de arroyos de flujo irregular y barrancos excavados bien sobre sustratos predominantemente calcáreos y suelos poco salinos, o bien (fig. 1B) sobre sustratos margoso-yesíferos y suelos de mayor salinidad.

C) Tarayales de cubetas endorreicas sobre suelos hipersalinos, compactos y de carácter asfixiante (fig. 1C).

La ordenación de los inventarios de comunidades de tarayal desarrolladas en estos tipos de medios permite reconocer tres unidades con rango de asociación: Tamaricetum gallicae para los tarayales subhalófilos (situaciones de tipo A y aspectos menos halófilos de las de tipo B); Agrostio-Tamaricetum canariensis para los tarayales mesohalófilos (situaciones de tipo B: subass. tamaricetosum canariensis) y ciertos tarayales hiperhalófilos (situaciones de tipo C: subass. inuletosum crithmoidis); y, por último, Suaedo-Tamaricetum boveanae para los tarayales hiperhalófilos (situaciones de tipo C) de la zona baja y oriental de la depresión. La primera de ellas se adscribe a la alianza subhalófila Tamaricion africanae y las dos últimas a la alianza Tamaricion boveano-canariensis, de exigencias halófilas más acentuadas. A continuación se describen estas unidades en detalle; la terminología bioclimática y biogeográfica sigue la última actualización de Rivas-Martínez (1987).

\section{Tamaricetum gallicae Br.-Bl. \& O. Bolòs 1957}

Asociación que comprende los tarayales dominados habitualmente por $T$. gallica que se desarrollan a lo largo de los lechos de inundación de los ríos (fig. 1A), así como de los cauces de ciertos arroyos excavados sobre sustratos predominantemente carbonatados.

En las riberas de los grandes ríos (Ebro, Segre, Cinca, etc.) estas comunidades se sitúan preferentemente sobre los depósitos fluviales de textura gruesa: gravosa, arenosa o areno-limosa; alternando hacia el cauce permanente, como primera galería forestal riparia, con las comunidades de saucedas (Salicetum neotrichae Br.-Bl. \& O. Bolòs 1957). Dicha situación puede ser considerada la estación primaria de la asociación. De modo general la vegetación de Tamaricion africanae reemplaza a las saucedas de Salicetalia purpureae y aún a otros bosques riparios de Populetalia en territorios cuyo clima -mesomediterráneo o más cálido- presenta largos períodos de aridez, con lo que las redes fluviales se tornan irregulares o incluso inoperantes. El efecto añadido de la salinización de las aguas, frecuente bajo tales condiciones climáticas si -como ocurre en la depresión del Ebro- abundan los sustratos suministradores de sales, resulta otro factor crítico para los bosques riparios caducifolios frente a los de Tamaricetalia. 
En los suelos aluviales estabilizados del lecho de inundación, la edafogénesis atenúa el efecto de la salinidad y se instala, como segunda galería forestal, el bosque de álamos (Rubio tinctori-Populetum albae Br.- Bl. \& O. Bolòs 1957). La degradación profünda de los medios propios de esta serie riparia -consecuencia, por ejemplo, de las labores de extracción de áridos- propicia el que los tarayales de Tamaricetum gallicae puedan desarrollarse, de manera secundaria, como comunidades sustituyentes de la alameda. La tercera galería forestal riparia, correspondiente a las olmedas, ha sido ampliamente transformada en las grandes vegas fluviales por la dedicación de su territorio potencial a cultivos hortícolas. En los cauces de flujo irregular no suele haber espacio ecológico ni siquiera para la instalación de la segunda galería forestal, de forma que el tarayal contacta directamente con las series climatófilas (Querceto rotundifoliae S. o Rhamno-Querceto cocciferae S.).

En la tabla 1 se aportan 12 inventarios que muestran la composición florística de la asociación. Destaca la dominancia, en la mayoría de los casos, de $T$. gallica frente a las restantes especies del género. Los inventarios 3-5 pueden asignarse a la subasociación tamaricetosum africanae O. Bolòs 1967, si bien el significado ecológico de la misma nos parece más ligado a caracteres edáficos texturales (suelos con gran proporción de gravas o quijarros) que, como había sugerido Bolòs (1967: 51), a un grado menor de halofilia. En la depresión del Ebro es frecuente la coexistencia de $T$. canariensis con las especies antedichas, lo que confirma lo postulado por Izco, Fernández-González \& Molina (1984: 381) acerca de la amplitud ecológica y carácter fitosociológico de este taray.

En la tabla sintética final (tabla 4) se aprecia que el cortejo diferencial de Tamaricetum gallicae comprende diversos táxones característicos de los órdenes Populetalia y Prunetalia spinosae, lo que denota el carácter subhalófilo de la asociación en el contexto del orden Tamaricetalia. Es igualmente destacable la mayor riqueza florística de esta asociación frente a las de las alianzas Tamaricion boveanocanariensis (número medio de táxones/inventario de 12,9 en nuestra tabla y 14 en la de Braun-Blanquet \& Bolòs (1957: tb. 40), frente a 6,7 en Agrostio-Tamaricetum canariensis y 7 en Suaedo-Tamaricetum boveanae), lo que pone de manifiesto el efecto selectivo del estrés salino.

El inventario 9 fue realizado en la localidad del lectotipo de la asociación (Gelsa, Zaragoza), designado por Izco, Fernández-González \& Molina (1984: 387). La mayor abundancia y grado de presencia de Tamarix gallica frente a T. canariensis, tanto en la localidad típica de la asociación como en el conjunto de los inventarios referibles a la misma, no apoyan, de cuerdo con el artículo 43 del CPN, la corrección nomenclatural ("Tamaricetum canariensis"). sugerida por O. Bolòs (1973: 297).

Los inventarios de la tabla 1 se han ordenado de acuerdo con lo que parece un incremento progresivo del carácter halófilo de las comunidades, detectado por el aumento de los valores de cobertura/abundancia de Tamarix canariensis, paralelo al de algunos táxones acompañantes con ciertas exigencias salinas, como Atriplex halimus o Lygeum spartum, y a la disminución recíproca de las diferenciales de Querco-Fagetea. Las variaciones florísticas resultan, sin embargo, demasiado graduales como para dividir la asociación en otros sintáxones subordinados. 


\section{Agrostio stoloniferae-Tamaricetum canariensis Cirujano 1981}

Asociación representada por tarayales de barrancos excavados en sustratos margoso-yesíferos, sobre suelos de mayor salinidad que en el caso anterior (fig. 1b). La dominancia en el estrato fanerofítico corresponde casi exclusivamente a Tamarix canariensis, con débiles presencias de T. gallica en situaciones de halofilia moderada. Paralelamente tiene lugar la desaparición de los elementos de Querco-Fagetea que diferencian a là asociación precedente, simultaneada con la entrada de un cierto número de halófitos entre los que destaca, por su abundancia y fidelidad, Suaeda vera var. braun-blanquetii, característica principal de la asociación fruticosa halófila Suaedetum braun-blanquetii Br.-Bl. \& O. Bolòs 1957 corr. (Suadetum brevifoliae Br.-Bl. \& O. Bolòs 1957; véase Apéndice florístico), que suele desarrollarse en los claros o la vecindad de estos tarayales. Además, Atriplex halimus tiene un grado de presencia notoriamente más elevado que en la asociación anterior.

En las cubetas endorreicas de algunas comarcas aragonesas interiores (Cinco Villas y Somontano) se encuentran tarayales de Tamarix canariensis acompañados de un mayor número de plantas halófilas, como Inula crithmoides, Arthrocnemum macrostachyum y Puccinellia fasciculata. Tal circunstancia permite reconocer una subasociación de mayores exigencias en cuanto a la salinidad, diferenciada por dichos elementos, y que denominamos inuletosum crithmoidis subass. nova (tabla 2: inv. 2-13; holotipo: inv. 13). Ocasionalmente, esta subasociación puede reconocerse también en ciertos tramos de algunos arroyos o barrancos en los que haya tenido lugar una acumulación local de sales (inv. 10 y 13).

La asociación en conjunto parece poseer una distribución mesomediterránea castellano-maestrazgo-manchega y aragonesa. La tabla original de Cirujano (1981:228, tb. 23), de procedencia manchega, revela algunas diferencias florísticas con respecto a la nuestra (véase tabla 4), siendo las más notables la ausencia de Suaeda braun-blanquetii y la nutrida presencia de Atriplex hastata; la ausencia de otros táxones de amplia distribución puede atribuirse al bajo número de especies por inventario que posee dicha tabla. Sin embargo, la dominancia de Tamarix canariensis en el estrato arbóreo es común en ambas, al igual que la falta total de elementos transgresivos de Populetalia y Prunetalia spinosae. Aunque Suaeda no aparezca en sus inventarios, las indicaciones ecológicas de Cirujano (1.c.) y la tabla de comunidades manchegas de Suaeda brevifolia (=Suaeda braun-blanquetii aportada por Castroviejo \& Cirujano (1980:149), con localidades coincidentes con las de inventarios de Agrostio-Tamaricetum canariensis, permiten inferir la vecindad de dicha planta con respecto a los tarayales descritos por Cirujano. Recíprocamente, a la ausencia de Atriplex hastata en la tabla 2 tampoco debe atribuírsele valor diferencial, por tratarse de un taxon que alcanza igualmente la depresión del Ebro (Br.-Bl. \& O. Bolòs, 1957: tabla 18, etc.).

La diferenciación de la subass. atriplicetosum halimi propuesta por Cirujano (op.cit.) resulta difícilmente mantenible en el valle del Ebro, donde la presunta especie diferencial -característica de las comunidades leñosas nitrófilas de SalsoloPeganetalia- tiene una amplia repartición que desborda los criterios ecológicos mantenidos por dicho autor.

La discriminación entre las asociaciones Tamaricetum gallicae y AgrostioTamaricetum canariensis no excluye la posibilidad de algunas situaciones edáficas 
y/o hidrológicas intermedias. Así, en la baja depresión del Ebro, de rigores xéricos más pronunciados, $T$. canariensis cobra mayor pujanza y puede llegar a ser el elemento dominante en cauces de flujo muy irregular aunque con bajos niveles de salinidad, como el ilustrado por el inv. 1 de la tabla 2 ; y viceversa, cauces de flujo importante excavados en terrenos más o menos yesíferos pueden albergar tarayales cuya composición florística encaja mejor en Tamaricetum gallicae (caso de los inv. 6 y $10-12$ de la tabla 1$)$.

3. Suaedo braun-blanquetii-Tamaricetum boveanae (Izco, FernándezGonzález \& Molina 1984) ass. nova

Syn.: Inulo crithmoidis-Tamaricetum boveanae subas. limonietosum latibracteati Izco, Fernández-González \& Molina 1984 (op. cit.: 392, tb. 2: inv. 11-14; holotipo: inv. 13)

Elevamos a asociación el sintaxon monegrino propuesto por Izco, FernándezGonzález \& Molina (1984). Entonces, a pesar de las apreciables divergencias florísticas que se observaban entre la asociación murciano-almeriense InuloTamaricetum boveanae y la única comunidad monegrina conocida de Tamarix boveana (Salada de Chiprana), el carácter de irradiación muy localizada atribuible a ésta aconsejaba un estatus subordinado. Actualmente, tras comprobar que los tarayales de $T$. boveana alcanzan una distribución mucho más amplia en la baja depresión aragonesa, parece conveniente asignarles rango de asociación independiente. Como diferenciales de Suaedo-Tamaricetum boveanae frente a Inulo-Tamaricetum boveanae pueden utilizarse (véase tabla 4): Suaeda vera var. braun-blanquetii, Puccinellia fasciculata, Limonium latibracteatum, Elymus repens, etc; y, en sentido inverso: Halimione portulacoides, Sarcocornia fruticosa, Suaeda vera, Suaeda pruinosa, Limonium eugeniae, etc.

Las comunidades de Suaedo-Tamaricetum boveanae resultan florísticamente próximas a las que hemos descrito como Agrostio-Tamaricetum canariensis inuletosum crithmoidis. Aparte de la ausencia en la segunda de Tamarix boveana y la rareza en la primera de Inula crithmoides y Atriplex halimus, se aprecia una interesante segregación geográfica (véase mapa 1) y bioclimática entre ambas. Suaedo-Tamaricetum se circunscribe a la parte baja y oriental de la depresión (Monegros y Desierto de Calanda), precisamente la más árida, térmica y continental (Loidi \& al., 1990); en tanto que ambientes endorreicos similares de las comarcas de Cinco Villas y Somontano, algo más frescas (mesomediterráneo medio o superior) y lluviosas (semiárido superior/seco inferior), son ocupadas por las comunidades de $T$. canariensis.

Respecto a la variabilidad dentro de la asociación, a lo ya expuesto por Izco \& al. (1984) se puede añadir la pauta marcada por el ingreso de Arthrocnemum macrostachyum en los niveles más bajos y, por consiguiente, de inundación más prolongada de las saladas. Esta variación, similar a la de otras asociaciones de la alianza, se ha detectado en la salada de Codo (inventarios 5 y 6).

Las poblaciones monegrinas y bajoaragonesas de $T$. boveana evocan una distribución pretérita más importante, ahora en trance de regresión por la puesta en cultivo de nuevas áreas y la desecación de diversas lagunas endorreicas. Así, junto a la mermada pero importante población de la salada de Chiprana (Izco \& al., 1984) y 


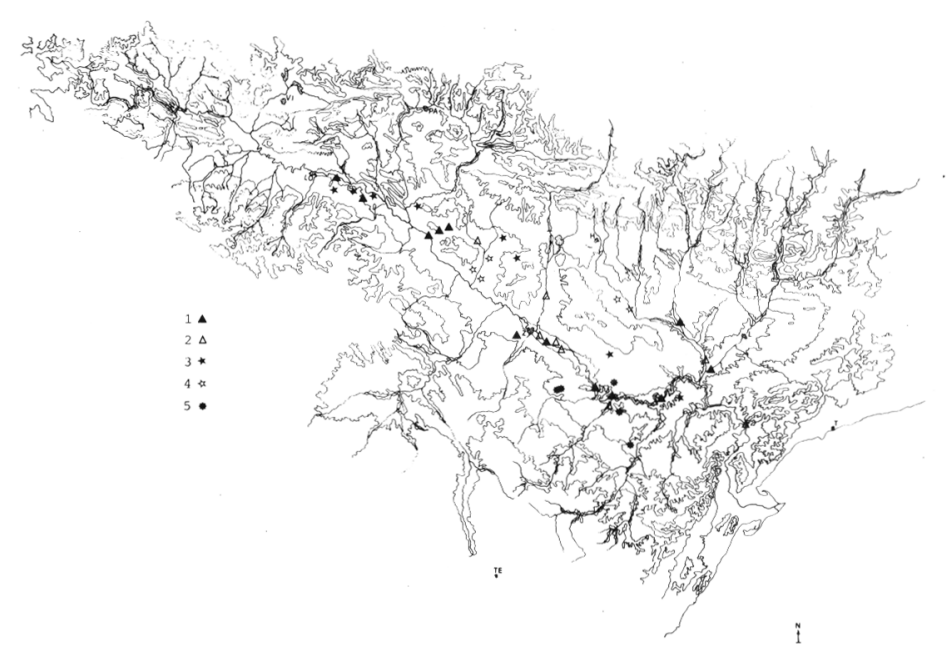

Mapa 1.- Localización de los inventarios en el valle del Ebro. 1-2: Tamaricetum gallicae (1: tabla 1, 2: otras localidades de Braun-Blanquet \& Bolòs, 1957). 3: Agrostio-Tamaricetum canariensis tamaricetosum. 4: Agrostio-Tamaricetum canariensis inuletosum. 5: SuaedoTamaricetum boveanae.

las aún más extensas del saladar de Codo -donde se localiza la mayor masa forestal peninsular de T. boveana que conocemos- este taray se halla al borde de la extinción en la salada de Alcañiz. En la plataforma de Bujaraloz, al norte del Ebro, donde se ubican numerosas lagunas saladas, al menos en las visitadas ha desaparecido todo vestigio de vegetación arbórea freatófila. En este sentido, suscribimos la inquietud manifestada por Blanché \& Molero (1988: 297) ante la amenaza que los planes de regadío en proyecto pueden suponer para los extraordinariamente frágiles ecosistemas de los saladares aragoneses.

\section{ES QUEMA S I N T A X ONOMICO}

NERIO-TAMARICETEA Br.-Bl. \& O. Bolòs 1957

+ Tamaricetalia africanae Br.-Bl. \& O. Bolòs 1957 em. Izco, F. Fernández-González \& A. Molina 1984

* Tamaricion africanae Br.-Bl. \& O. Bolòs 1957

1. Tamaricetum gallicae $\mathrm{Br}$--Bl. \& O. Bolòs 1957

1a. subas. tamaricetosum gallicae

1b. subas. tamaricetosum africanae O. Bolòs 1967

* Tamaricion boveano-canariensis Izco, F. Fernández-González \& A. Molina 1984

2. Agrostio stoloniferae-Tamaricetum canariensis Cirujano 1981

2a. subass. tamaricetosum canariensis

2b. subass. inuletosum crithmoidis nova

3. Suaedo braun-blanquetii-Tamaricetum boveanae (Izco, F. Fernández-González \& A. Molina 1984) ass. nova 
A P E N D I C E F L O R I S T I C O. La nomenclatura florística se atiene a la adoptada en Flora Europaea 1-5 (Tutin \& al. (eds.), Cambridge Univ. Press, 1964/1980) y MedChecklist 1, 3-4 (Greuter, Burdet \& Long, Conserv. Jard. Bot. Genève, 1984/1989), con la excepción de algunos táxones infraespecíficos que aparecen abreviados binomialmente en las tablas, y de los casos siguientes: Crepis vesicaria subsp. taraxacifolia (Thuill.) Thell., Parietaria judaica L., Salix neotricha Görz, Limonium latibracteatum Erben. Suaeda vera var. o subsp. brevifolia auct. iber., non S. fruticosa var. brevifolia Moq., debe llamarse Suaeda vera var. braun-blanquetii Pedrol \& Castroviejo, Anales Jard. Bot. Madrid 45(1): 95. 1988. Para las determinaciones de las especies del género Tamarix se han seguido los criterios de Baum (1978).

\section{B I B L I O G R A F I A}

BAUM, B. -1978- The genus Tamarix. Israel Acad. Sci. Humanities, 1-209.

BLANCHE, C. \& MOLERO, J. -1988- Las cubetas arreicas al sur de Bujaraloz (Valle del Ebro). Contribución a su estudio fitocenológico. Lazaroa, 9:277-299.

BRAUN-BLANQUET, J. \& BOLOS, A. -1957- Les groupements végétaux du bassin moyen de l'Ebre et leur dynamisme. Anales Estac. Exper. Aula Dei, 5(1-4):1-266.

BOLOS, O. -1967-Comunidades vegetales de las comarcas próximas al litoral situadas entre los ríos Llobregat y Segura. Mem. Real Acad. Ci. Art. Barcelona, 38(1):1-269.

BOLOS, A. -1973- La vegetación de la Serreta Negra de Fraga. Mem. Real Acad. Ci. Art. Barcelona, 42:269-313.

CASTROVIEJO, S. \& CIRUJANO, S. -1980- Sarcocornietea en La Mancha (España). Anales Jard. Bot. Madrid 37(1):143-154.

CIRUJANO, S. -1981- Las lagunas manchegas y su vegetación, II. Anales Jard. Bot. Madrid, 38(1):187-232.

IZCO, J., FERNANDEZ-GONZALEZ, F. \& MOLINA, A. -1984- El orden Tamaricetalia Br.B1. \& O. Bolòs 1957 y su ampliación con los tarayales hiperhalófilos. Doc. Phytosociol., n.s. 8:377-392.

LOIDI, J., FERNANDEZ-GONZALEZ, F. \& MOLINA, A. -1990- Pródromo de las comunidades de matorral de la depresión del Ebro. Inst. Est. Turolenses (en prensa).

RIVAS MARTINEZ, S. -1987- Memoria del mapa de series de vegetación de España 1:400.000. I.C.O.N.A. Madrid.

(Aceptado para su publicación el 23 de enero de 1990)

Dirección de los autores: F. FERNANDEZ-GONZALEZ: Departamento de Biología Vegetal II (Botánica), Facultad de Farmacia, Universidad Complutense, E-28040 Madrid. A. MOLINA: Departamento de Biología Vegetal I, Facultad de Biología, Universidad Complutense, E-28040 Madrid. J. LOIDI: Laboratorio de Botánica, Departamento de Biología Vegetal y Ecología, Facultad de Ciencias, Universidad del País Vasco, Ap. 644, E-48080 Bilbao. 

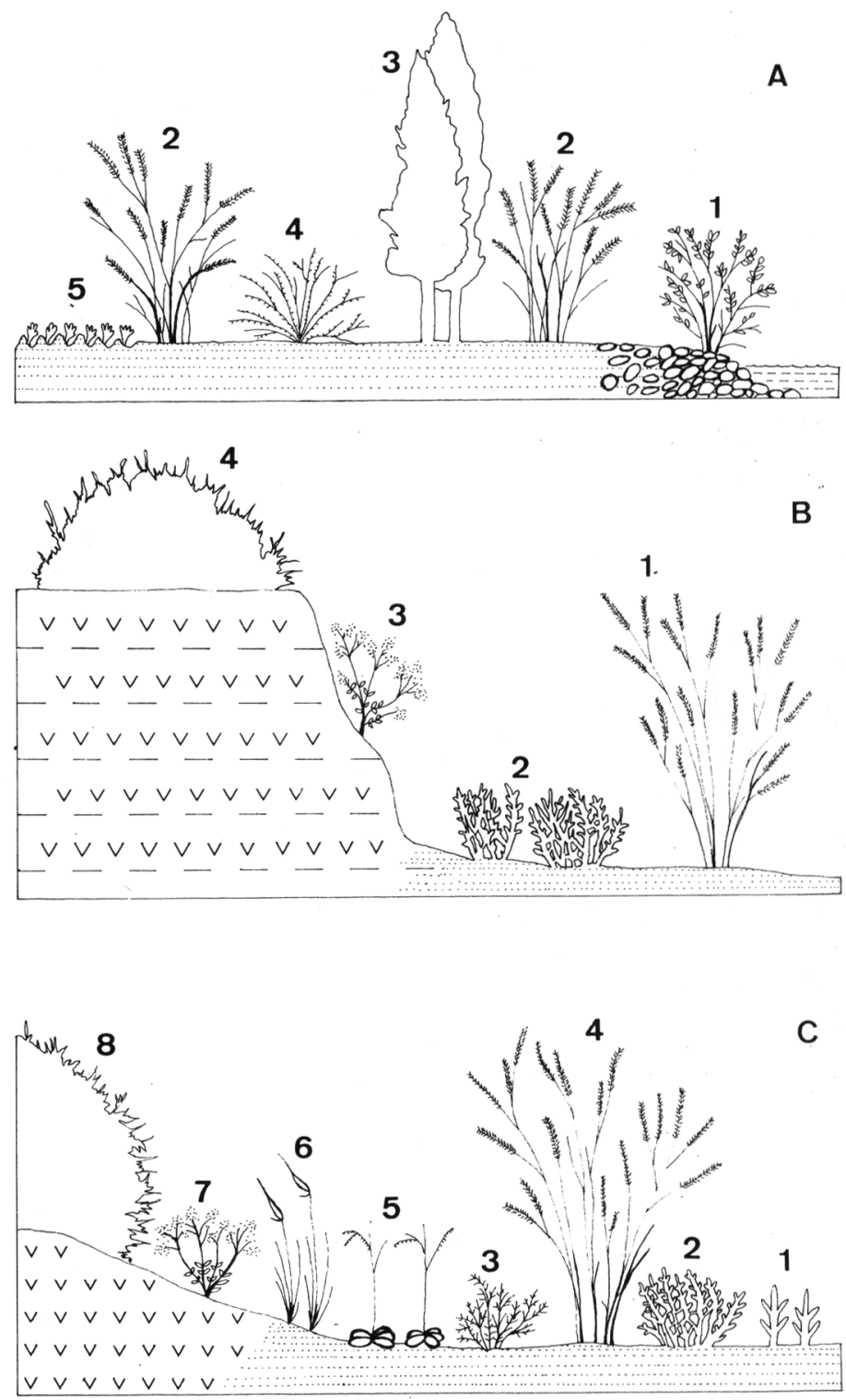

Figura 1.- A: El Burgo de Ebro, ribera del Ebro. B: barranco de Las Bardenas. C: saladar de Codo. A1: Salicetum neotrichae. A2: Tamaricetum gallicae. A3: Rubio-Populetum albae. A4: Pruno-Rubion ulmifolii. A5: cultivos hortícolas de vega. B1: Agrostio-Tamaricetum canariensis. B2, C3: Suaedetum braun-blanquetii. B3, C7: Gypsophilenion hispanicae. B4, C8: Rhamno-Quercetum cocciferae. C1: Salicornietum ramosissimae. C2: Suaedetum braun-blanquetii arthrocnemetosum macrostachyi. C4: Suaedo-Tamaricetum boveanae. C5: Limonietum latibracteati. C6: Agropyro cristati-Lygeetum. 


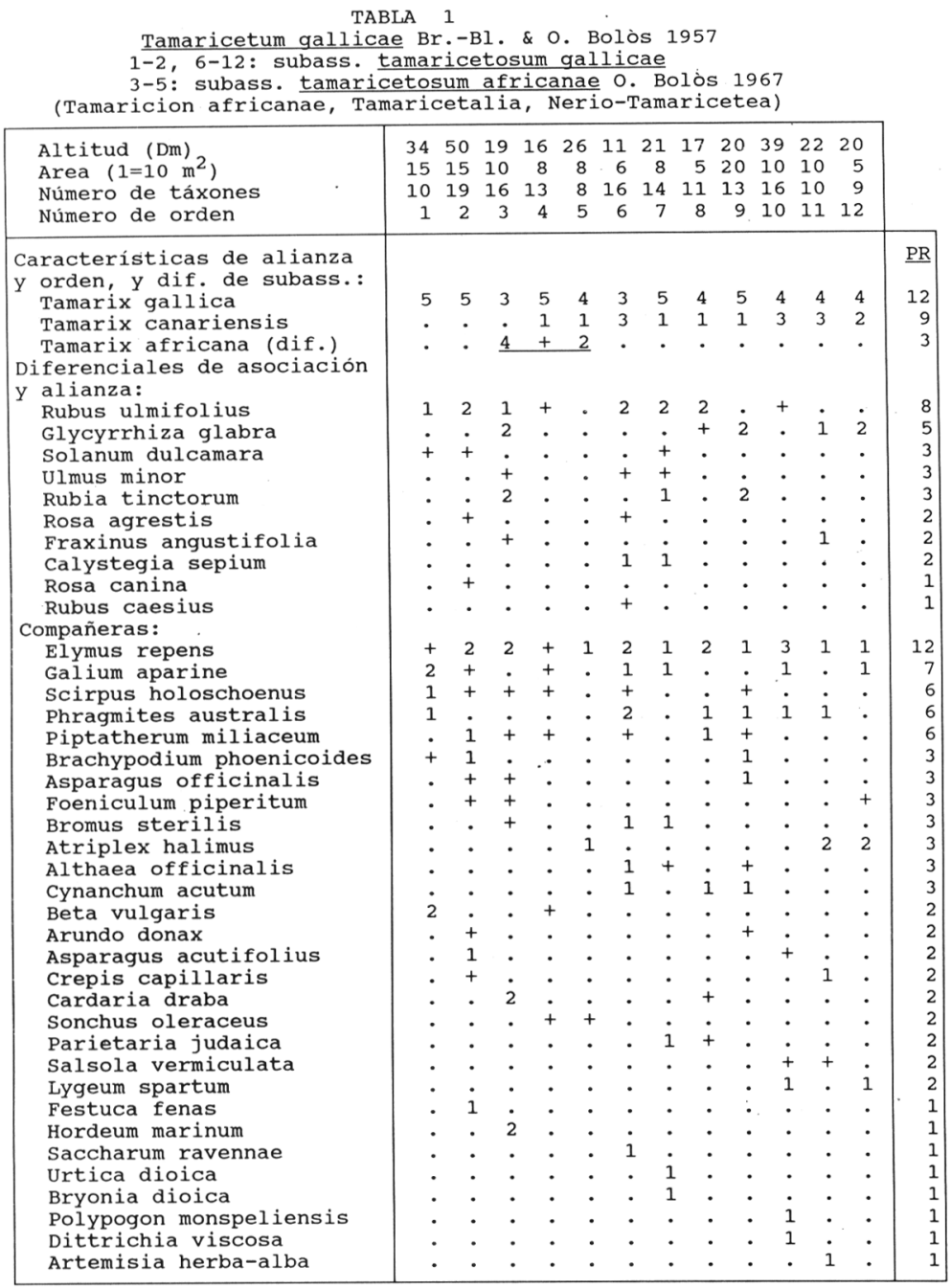

otros táxones con índices inferiores a 1: en 1: Cirsium monspessulanum; en 2: Juncus maritimus, Genista scorpius, Lycium cf. europaeum; en 3: Convolvulus arvensis, Rumex crispus; en 4: Juncus acutus, Polygonum equisetiforme, Hordeum leporinum; en 5: Silybum marianum; en 6 : Cardus bourgaeanus; en 9: Saponaria officinalis; en 0 : Melica magnotit, pentaphyllum.

Tabla 1. Localidades, coordenadas UTM y fechas de los inventarios: 1, LO: Ausejo-Logroño, desviación a Arrubal; charca no salobre (20TWM69, 90688/1). 2, LO: Arnedo-Calahorra; barranco sobre calizas duras (30TWM78, 90688/4). 3, Z: El Burgo de Ebro, ribera del Ebro (30TXM80, 120688/7). 4, Z: Caspe, ribera del embalse (31TBF57, 110688/5). 5, NA: Bardena Negra; rambla de cantos calcáreos (30TXM26, 90688/12). 6, L: Serós, río Segre (31TBF89, 110688/2). 7, NA: Tudela, ribera del Ebro (30TXM15, 90688/11). 8, Z: Escatrón, ribera del Ebro (30TYL27, 110688/7). 9, Z: Gelsa, margen izquierda del Ebro (30TYL18, 120688/2). 10, Z: La Peñaza, entre Zaragoza y La Muela; barranco (30TXM61, 120688/8). 11, NA: Bardena Real; barranco (30TXM16, 90688/10). 12, HU: Alcolea de Cinca (31TBG52, 110688/1). 
TABLA 2

Agrostio stoloniferae-Tamaricetum canariensis Cirujano 1981

1-8: subass. tamaricetosum canariensis

9-13: subass. inuletosum crithmoidis nova

(Tamaricion boveano-canariensis, Tamaricetalia, Nerio-Tamaricetea)

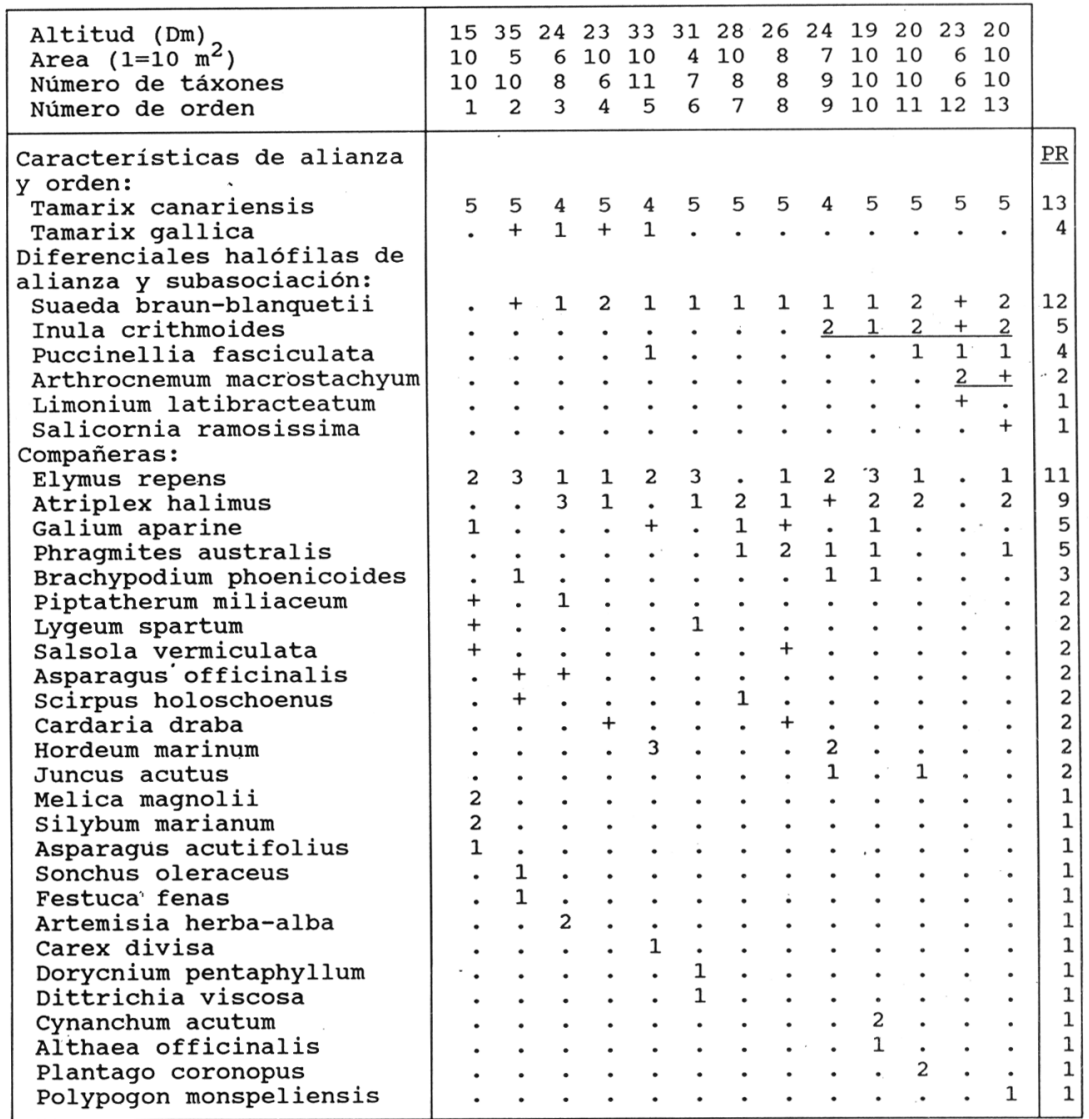

otros táxones con indices inferiores a 1: en 1: Convolvulus arvensis; en 2: Cirsium monspessulanum; en 5: Juncus maritimus, Crepis taraxacifolia, Medicago arabica; en 7: Bromus sterilis, Urtica dioica; en 11: Juncus gerardi i, Equisetum ramosissimum.

Tabla 2. Localidades, coordenadas UTM y fechas de los inventarios: 1, Z: Caspe-Mequinenza; pequeña rambla (31TBF67, 110688/4). 2, LO: Ausejo; barranco con arroyo (30TWM68, 90688/2). 3, NA: Bardenas Reales; barranco (30TXM27, 90688/8). 4, Z: Bujaraloz-Gelsa; salitral (30TYL19, 120688/1). 5, LO: Alcanadre; cubeta endorreica (30TWM69, 90688/3). 6, LO: Calahorra; salitral (30TWM88, 90688/5). 7, Z: Ejea de los Cabelleros, río Rigüel (30TXM46, 90688/4). 8, Z: Castejón de Valdejasa; barranco (30TXM65, 100688/6). 9, HU: Lalueza; acequia de drenaje (30TYM23, 100688/10). 10, HU: Lalueza-Sariñena, río Flumen (30TYM33, 100688/11). 11, Z: Ejea de los Caballeros-Tauste; salitral (30TXM56, 100688/1). 12, Z: Cerca de Tauste; salitral (30TXM54, 100688/5). 13, Z: Tauste, barranco de Mira (30TXM45, 100688/2). 
TABLA 3

Suaedo braun-blanquetii-Tamaricetum boveanae ass. nova (Tamaricion boveano-canariensis, Tamaricetalia, Nerio-Tamaricetalia)

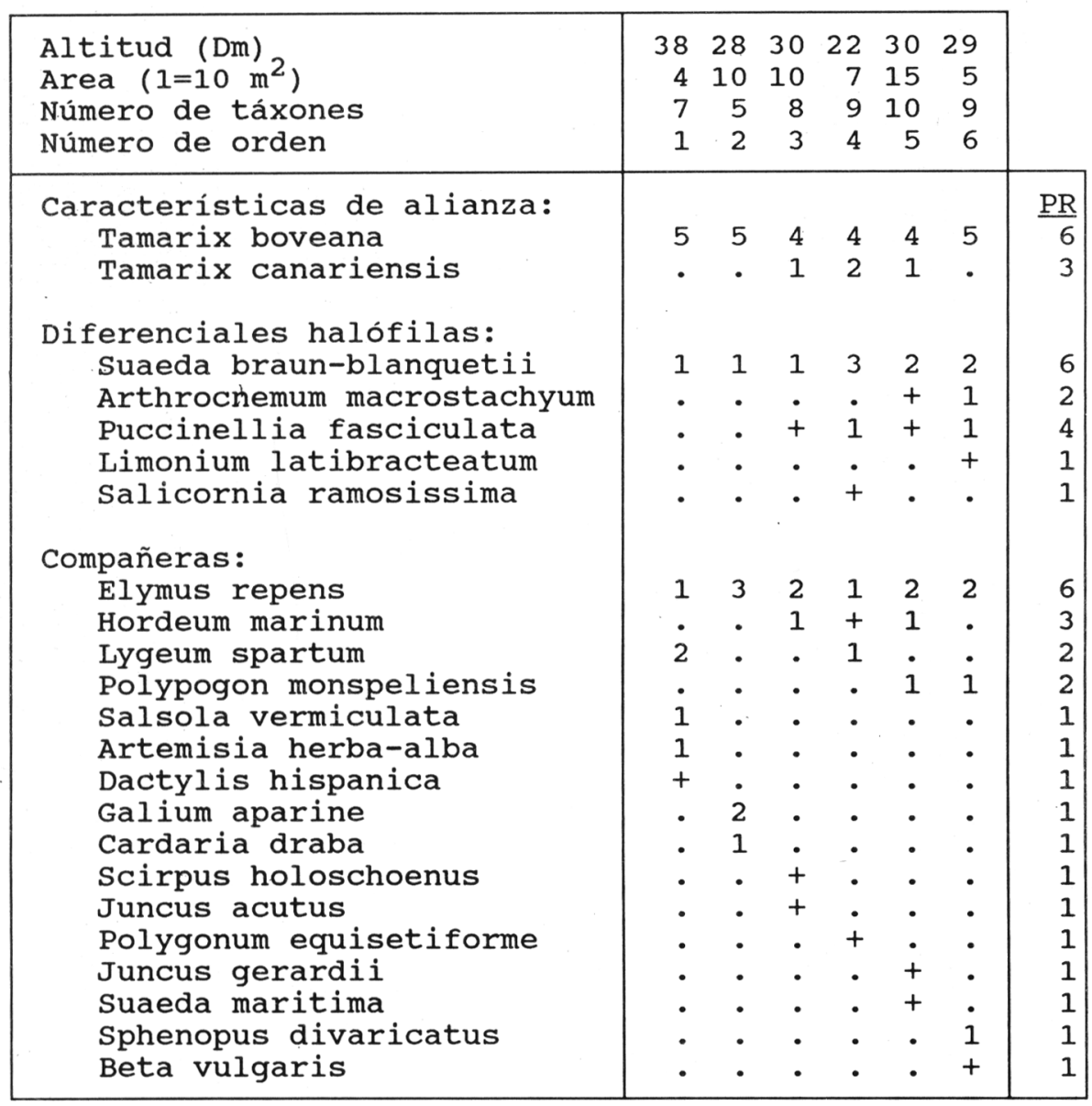

Tabla 3. Localidades, coordenadas UTM y fechas de los inventarios: 1, TE: La Salada de Alcañiz (30TYL34, 110688/6). 2, Z: Escatrón-Bujaraloz, barranco de drenaje de salitral (30TYL28, 110688/8). 3-6, Z: Codo-Belchite, salitral (30TXL98, 120688/4, 3, 5, 6). 
TABLA 4

\begin{tabular}{|c|c|c|c|c|c|c|c|}
\hline Número de inventarios & & 121 & & 8 & & 101 & \\
\hline Número de orden & 1 & 2 & 3 & 4 & & 6 & 7 \\
\hline (n) & $\cdots$ & & & & & & $\cdots$ \\
\hline $\begin{array}{l}\text { Características de alianzas } \\
\text { y orden }\end{array}$ & & & & & & & \\
\hline Tamarix gallica & 5 & 5 & . & 3 & - & - & $\cdot$ \\
\hline Tamarix africana & 1 & 2 & $\cdot$ & - & . & - & - \\
\hline Tamarix canariensis & . & 4 & 5 & 5 & 5 & 2 & 2 \\
\hline Tamarix boveana & · & $\cdot$ & $\cdot$ & $\cdot$ & - & 5 & 5 \\
\hline $\begin{array}{l}\text { Diferenciales de Iamaricion } \\
\text { africanae }\end{array}$ & & & & & & & \\
\hline Glycyrrhiza glabra & 3 & 3 & $\cdot$ & $\cdot$ & $\cdot$ & $\cdot$ & $\cdot$ \\
\hline Rubus ulmifolius & 1 & 4 & $\cdot$ & $\cdot$ & $\cdot$ & - & $\cdot$ \\
\hline Solanum dulcamara & 1 & 2 & $\cdot$ & · & . & $\cdot$ & $\cdot$ \\
\hline Calystegia sepium & 1 & 1 & $\cdot$ & $\cdot$ & • & $\cdot$ & $\cdot$ \\
\hline Lycopus europaeus & 1 & $\cdot$ & $\cdot$ & $\cdot$ & $\cdot$ & $\cdot$ & $\cdot$ \\
\hline Populus alba & 1 & $\cdot$ & $\cdot$ & $\cdot$ & $\cdot$ & $\cdot$ & $\cdot$ \\
\hline Populus nigra & 1 & $\cdot$ & $\cdot$ & $\cdot$ & $\cdot$ & $\cdot$ & $\cdot$ \\
\hline Salix purpurea & 1 & $\dot{0}$ & $\cdot$ & $\cdot$ & $\cdot$ & $\cdot$ & $\cdot$ \\
\hline Ulmus minor & $\cdot$ & 2 & $\cdot$ & $\cdot$ & $\cdot$ & $\cdot$ & $\cdot$ \\
\hline Rubia tinctorum & $\cdot$ & 2 & $\cdot$ & $\cdot$ & $\cdot$ & $\cdot$ & $\cdot$ \\
\hline Rubus caesius & $\cdot$ & 1 & $\cdot$ & $\cdot$ & $\cdot$ & $\cdot$ & $\cdot$ \\
\hline Fraxinus angustifolia & $\cdot$ & 1 & $\cdot$ & $\cdot$ & $\cdot$ & $\cdot$ & $\cdot$ \\
\hline Bryonia dioica & $\cdot$ & 1 & $\cdot$ & $\cdot$ & $\cdot$ & $\cdot$ & $\cdot$ \\
\hline Rosa agrestis & $\cdot$ & 1 & $\cdot$ & $\cdot$ & $\cdot$ & $\cdot$ & $\cdot$ \\
\hline Rosa canina & - & 1 & $\cdot$ & $\cdot$ & $\cdot$ & $\cdot$ & $\cdot$ \\
\hline $\begin{array}{l}\text { Diferenciales de Tamaricion } \\
\text { boveano-canariensis }\end{array}$ & & & & & & & \\
\hline Aeluropus littoralis & $\cdot$ & $\cdot$ & 2 & - & $\cdot$ & 1 & 1 \\
\hline Suaeda braun-blanqueti i & $\cdot$ & $\cdot$ & $\cdot$ & 5 & 5 & 5 & $\cdot$ \\
\hline Puccinellia fasciculata & $\cdot$ & $\cdot$ & $\cdot$ & 1 & 3 & 2 & - \\
\hline Inula crithmoides & - & $\cdot$ & $\cdot$ & - & 5 & 1 & 3 \\
\hline Arthrocnemum macrostachyum & $\cdot$ & $\cdot$ & $\cdot$ & $\cdot$ & 2 & 1 & 3 \\
\hline Limonium latibracteatum & $\cdot$ & $\cdot$ & $\cdot$ & $\cdot$ & 1 & 2 & - \\
\hline Salicornia ramosissima & $\cdot$ & $\cdot$ & $\cdot$ & $\cdot$ & 1 & 1 & - \\
\hline Atriplex hastata & 1 & $\cdot$ & 4 & $\cdot$ & . & - & $\cdot$ \\
\hline Gypsophila tomentosa & $\cdot$ & $\cdot$ & 1 & $\cdot$ & $\cdot$ & $\cdot$ & $\cdot$ \\
\hline Sonchus maritimus & $\cdot$ & $\cdot$ & 1 & $\cdot$ & $\cdot$ & $\cdot$ & $\cdot$ \\
\hline Suaeda maritima & $\cdot$ & $\cdot$ & $\cdot$ & $\cdot$ & $\cdot$ & 1 & $\cdot$ \\
\hline Sarcocornia fruticosa & $\cdot$ & $\cdot$ & $\cdot$ & $\cdot$ & $\cdot$ & $\cdot$ & 5 \\
\hline Halimione portulacoides & $\cdot$ & $\cdot$ & $\cdot$ & $\cdot$ & $\cdot$ & - & 4 \\
\hline Limonium eugeniae & $\cdot$ & $\cdot$ & $\cdot$ & $\cdot$ & $\cdot$ & $\cdot$ & 4 \\
\hline Suaeda vera \& pruinosa & $\cdot$ & $\cdot$ & $\cdot$ & $\cdot$ & $\cdot$ & - & 4 \\
\hline Spergularia marginata & . & $\cdot$ & $\cdot$ & $\cdot$ & $\cdot$ & . & 2 \\
\hline Atriplex glauca & $\cdot$ & $\cdot$ & $\cdot$ & $\cdot$ & $\cdot$ & $\cdot$ & 1 \\
\hline Frankenia corymbosa & $\cdot$ & $\cdot$ & $\cdot$ & $\cdot$ & $\cdot$ & $\cdot$ & 1 \\
\hline Lycium intricatum & $\cdot$ & - & $\cdot$ & - & $\cdot$ & - & 1 \\
\hline
\end{tabular}

\begin{tabular}{|c|c|c|c|c|c|c|c|}
\hline \multirow{2}{*}{\begin{tabular}{|l|} 
Número de orden \\
Otras compañeras:
\end{tabular}} & \multicolumn{7}{|c|}{$\begin{array}{lllllll}1 & 2 & 3 & 4 & 5 & 6 & 7\end{array}$} \\
\hline & \\
\hline Phragmi tes austral is & 1 & 3 & 3 & 2 & 3 & 1 & 5 \\
\hline Atriplex halimus & 1 & 2 & 2 & 4 & 4 & 1 & 1 \\
\hline Scirpus holoschoenus & 1 & 3 & 1 & 2 & . & 1 & - \\
\hline Juncus maritimus & 1 & 1 & 1 & 1 & . & 1 & . \\
\hline Elymus repens & 4 & 5 & . & 5 & 4 & 3 & . \\
\hline Hordeum marinum & 1 & 1 & . & 1 & 1 & 2 & . \\
\hline Althaea officinal is & 2 & 2 & 1 & $\cdot$ & 1 & . & . \\
\hline Brachypodium phoenicoides & 4 & 2 & . & 1 & 2 & . & - \\
\hline Artemisia herba-alba & 1 & 1 & . & 1 & . & 2 & $\cdot$ \\
\hline Cardaria draba & 1 & 1 & . & 2 & - & 1 & - \\
\hline Polypogon monspeliens is & - & 1 & 1 & . & 1 & 1 & . \\
\hline Gal ium aparine & - & 3 & . & 3 & 1 & 1 & - \\
\hline Lygeum spartum & - & 1 & . & 2 & . & 2 & 2 \\
\hline Juncus acutus & . & 1 & . & . & 2 & 1 & 4 \\
\hline Piptatherum miliaceum & 1 & 3 & - & 2 & . & . & . \\
\hline Dittrichia viscosa & 2 & 1 & $\cdot$ & 1 & . & . & $\cdot$ \\
\hline Asparagus officinalis & 1 & 2 & . & 2 & . & . & . \\
\hline Asparagus acutifolius & 1 & 1 & . & 1 & . & . & $\cdot$ \\
\hline Festuca fenas & 1 & 1 & $\cdot$ & 1 & . & . & . \\
\hline Cymanchum acutum & 1 & 2 & . & $\cdot$ & 1 & . & . \\
\hline Salsola vermiculata & $\cdot$ & 1 & $\cdot$ & 2 & $\cdot$ & 1 & $\cdot$ \\
\hline Saccharum ravennae & 3 & 1 & - & - & . & . & . \\
\hline Foeniculum piperitum & 1 & 2 & $\cdot$ & - & $\cdot$ & $\cdot$ & $\cdot$ \\
\hline Arundo donax & 1 & 19 & $\cdot$ & $\cdot$ & $\cdot$ & $\cdot$ & $\cdot$ \\
\hline Renunculus repens & 1 & 1 & - & $\cdot$ & $\cdot$ & - & . \\
\hline Rumex crispus & 1 & 1 & $\cdot$ & $\cdot$ & $\cdot$ & $\cdot$ & $\cdot$ \\
\hline Equisetum ramosissimum & 1 & · & $\cdot$ & - & 1 & - & - \\
\hline Imperata cylindrica & 2 & . & $\cdot$ & - & $\cdot$ & $\cdot$ & 1 \\
\hline Cirsium monspessulanum & . & 1 & - & 1 & . & $\cdot$ & . \\
\hline Doryenium pentaphyl lum & . & 1 & $\cdot$ & 1 & . & - & - \\
\hline Melica magnol $i i$ & $\cdot$ & 1 & $\cdot$ & 1 & - & - & . \\
\hline Urtica dioica & $\cdot$ & 1 & $\cdot$ & 1 & $\cdot$ & · & $\cdot$ \\
\hline Polygonum equisetiforme & . & 1 & . & $\cdot$ & $\cdot$ & 1 & - \\
\hline Sphenopus divaricatus & . & 1 & - & . & . & 1 & - \\
\hline Juncus gerardii & $\cdot$ & $\cdot$ & $\cdot$ & $\cdot$ & 1 & 1 & $\cdot$ \\
\hline Eupator ium camabinum & 1 & $\cdot$ & $\cdot$ & $\cdot$ & $\cdot$ & $\cdot$ & $\cdot$ \\
\hline Retama sphaerocarpa & 1 & $\cdot$ & - & $\cdot$ & $\cdot$ & . & - \\
\hline Rubia peregrina & 1 & $\cdot$ & . & . & . & $\cdot$ & - \\
\hline Saponaria officinal is & $\cdot$ & 1 & $\dot{0}$ & $\cdot$ & $\cdot$ & $\cdot$ & $\cdot$ \\
\hline Agrostis stolonifera & $\cdot$ & $\cdot$ & 3 & $\cdot$ & . & $\cdot$ & · \\
\hline Elymus curvifolius & . & · & 1 & $\cdot$ & . & . & . \\
\hline Thymelaea hirsuta & & & & & & & \\
\hline
\end{tabular}

Procedencia de los inventarios:

1-2: Iamaricetum gallicae. 1: Braun-Blanquet \& Bolòs, 1957: tb. 40 (excl. inv. 10); 2: tabla 1.

3-5: Agrostio-Tamaricetum canariensis. 3: Cirujano, 1981: 228, tb. 23; 4: tabla 2, inv. 1-8; 5: tabla 2, inv. 9-13.

6: Suaedo-Tamaricetum boveanae. 4 inv. de Izco \& al., 1984: tb. 2, inv. 11-14; y 6 inv. de la tabla 3.

7: Inulo-Tamaricetum boveanae. Izco \& al., 1984: tb. 2, inv. 1-10. 